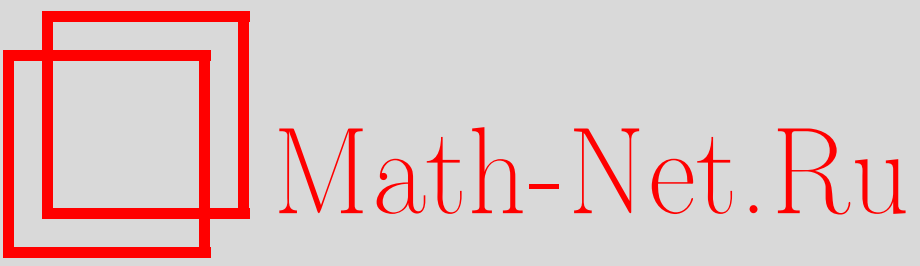

А. А. Пожарский, Полукристалл с сингулярным потенциалом в электрическом поле, ТМФ, 2006, том 146, номер 3, 410-428

DOI: https://doi.org/10.4213/tmf2044

Использование Общероссийского математического портала Math-Net.Ru подразумевает, что вы прочитали и согласны с пользовательским соглашением

http://www.mathnet.ru/rus/agreement

Параметры загрузки:

IP: 35.173 .219 .12

26 апреля 2023 г., 16:40:23 


\title{
ФИЗИКА
}

Том 146, № 3

март, 2006

(C) 2006 г.

\author{
А. А. Пожарский
}

\section{ПОЛУКРИСТАЛЛ С СИНГУЛЯРНЫМ ПОТЕНЦИАЛОМ В УСКОРЯЮЩЕМ ЭЛЕКТРИЧЕСКОМ ПОЛЕ}

\begin{abstract}
Изучается уравнение Шредингера, описывающее одномерное движение квантового электрона в периодическом кристалле, помещенном в ускоряющее электрическое поле. Описано асимптотическое поведение решений уравнения при больших значениях аргумента. На основе анализа полученных асимптотик указаны достаточно свободные условия на потенциал, при которых спектр соответствующего оператора чисто абсолютно непрерывен и заполняет всю вещественную ось.
\end{abstract}

Ключевые слова: уравнение Шредингера, асимптотическое решение, адиабатическое решение, точка поворота, абсолютно непрерывный спектр.

\section{1. ВВЕДЕНИЕ}

Рассмотрим одномерное движение квантового электрона в периодическом кристалле, помещенном в ускоряющее электрическое поле. Это движение описывается уравнением

$$
-\psi^{\prime \prime}-F(x) \psi+p(x) \psi=E \psi, \quad E \in \mathbb{R},
$$

на полуоси $0 \leqslant x<+\infty$. Будем предполагать, что потенциалы $p(x)$ и $F(x)$ удовлетворяют следующим условиям:

(A1) $p(x)$ - вещественная функция, $p(x+1)=p(x), p(x) \in L_{1, \mathrm{loc}}\left(\mathbb{R}_{+}\right), \int_{0}^{1} p(x) d x=0$;

(А2) $F(x)$ - вещественная функция, $F(x) \in C^{2}\left(\mathbb{R}_{+}\right), \quad F(0)=0, \quad F(+\infty)=+\infty$, существуют постоянные $\varepsilon, C_{1}, C_{2}$ и $C_{3}$ такие, что $C_{2} \geqslant C_{1}>0, C_{3} \geqslant 0$, $0<\varepsilon \leqslant 2, \quad C_{1} F^{(1-\varepsilon) / 2} \leqslant F^{\prime}(x) \leqslant C_{2} F^{(1-\varepsilon) / 2}, \quad\left|F^{\prime \prime}(x)\right| \leqslant C_{3} F^{-\varepsilon}$ при всех $x>0$.

Заметим, что функция $F(x)=x^{2 /(1+\varepsilon)}$ при $\varepsilon \in(0,2]$ удовлетворяет условию $(\mathrm{A} 2)$. Введем оператор в $L_{2}\left(\mathbb{R}_{+}\right)$вида

$$
H_{d}=-\frac{d^{2}}{d x^{2}}-F(x)+p(x)
$$

${ }^{*}$ Санкт-Петербургский государственный университет, Санкт-Петербург, Россия. E-mail: pozharsky@math.nw.ru 
с областью определения

$$
\operatorname{Dom}\left(H_{d}\right)=\left\{\varphi: \begin{array}{c}
\varphi^{\prime} \text { абсолютно непрерывна, } \varphi(0)=0, \\
\operatorname{supp} \varphi \text { ограничен, }-\varphi^{\prime \prime}+p \varphi \in L_{2}\left(\mathbb{R}_{+}\right)
\end{array}\right\} .
$$

ЗАмечАниЕ 1. Функция $-\varphi^{\prime \prime}+p(x) \varphi$ принадлежит $L_{2}\left(\mathbb{R}_{+}\right)$при $\varphi \in \operatorname{Dom}\left(H_{d}\right)$, но $\varphi^{\prime \prime}$ и $р \varphi$ по отдельности не обязательно принадлежат $L_{2}\left(\mathbb{R}_{+}\right)$. Однако они принадлежат $L_{1}\left(\mathbb{R}_{+}\right)$.

Оператор $H_{d}$ в существенном самосопряжен. Доказательство подобного факта для случая линейного потенциала $F(x)$ приведено в работе [1]. Доказательство в случае потенциала $F(x)$, удовлетворяющего условию (А2), проводится аналогично. Обозначим замыкание оператора $H_{d}$ через $H$.

В случае гладкого потенциала $p(x)$ уравнение (1.1) хорошо изучено. Приведем одно классическое утверждение, которое можно получить, используя результаты, приведенные в [2]. Пусть $p(x)$ - дважды непрерывно дифференцируемая функция, удовлетворяющая условию (A1), и $F(x)$ удовлетворяет условию $(\mathrm{A} 2)$, причем $\varepsilon<2$. Тогда существуют два линейно независимых решения $\psi_{+}$и $\psi_{-}$уравнения (1.1), допускающие при $x \rightarrow+\infty$ асимптотическое разложение вида

$$
\psi_{ \pm}(x)=\frac{1}{\sqrt[4]{F(x)}}\left\{\exp \left( \pm i \int_{x_{0}}^{x} \sqrt{E+F(t)-p(t)} d t\right)+o(1)\right\}
$$

В работах [3] было рассмотрено уравнение (1.1) на всей оси в случае гладкого периодического потенциала $p(x)$ и линейного потенциала $F(x)=x$. Показано, например, что если $p$ имеет две непрерывные производные, то оператор $H$ имеет чисто абсолютно непрерывный спектр, заполняющий всю вещественную ось (для уравнения (1.1) на положительной полуоси этот результат сохраняет силу).

Недавно в работах [4] была доказана абсолютная непрерывность спектра $H$ при более слабых условиях на периодический потенциал $p$ и при $F(x)=x$. Например, если $p$ имеет одну непрерывную производную, то оператор $H$ имеет чисто абсолютно непрерывный спектр, заполняющий всю вещественную ось (результат справедлив для уравнений вида (1.1) на всей оси $-\infty<x<\infty$ и на положительной полуоси $0 \leqslant x<\infty)$. Этот результат сформулирован в терминах коэффициентов Фурье периодического потенциала $p$ и является частным случаем теоремы 2 (при $\varepsilon=1$ ) настоящей работы.

Изучению абсолютно непрерывного спектра оператора $H$ с линейным потенциалом $F$ и локально интегрируемым потенциалом $p$ посвящена работа [1]. В ней указан широкий класс потенциалов $p$, для которых абсолютно непрерывная компонента спектра оператора $H$ заполняет всю вещественную ось. Этот класс включает, например, потенциалы вида

$$
p(x)=p_{0}(x)+|x-a|^{-b}, \quad x \in[0,1],
$$

где $p_{0}$ имеет одну непрерывную производную и $a \in[0,1], b \in(0,1)$. Для остальных $x$ потенциал $p$ продолжается периодическим образом. 
Отметим работу [5], в которой рассматривался случай линейного потенциала $F$ и периодического потенциала вида

$$
p(x)=V \sum_{n=-\infty}^{+\infty} \delta^{\prime}(x-n) .
$$

Доказано, что в этом случае спектр оператора $H$ не содержит абсолютно непрерывной компоненты.

По-видимому, одним из наиболее интересных и вместе с тем трудных случаев является оператор $H$ с линейным потенциалом $F(x)=F_{1} x$ и периодическим потенциалом вида

$$
p(x)=V \sum_{n=-\infty}^{+\infty} \delta(x-n)
$$

В работе [6] было проведено описание асимптотик решений уравнения (1.1) при больших значениях аргумента, а также показано, что природа спектра оператора $H$ существенно зависит от значения параметра $\pi^{2} / F_{1}$. При этом, однако, в настоящее время структура спектра данного оператора остается практически не изученной.

Работа [7] посвящена изучению линейного потенциала $F$ и случайного сингулярного потенциала $p$, заданного в виде

$$
p(x)=\sum_{n=-\infty}^{+\infty} V_{n} \delta(x-n),
$$

где коэффициенты $V_{n}$ случайны, одинаково распределены, и их среднее значение равно нулю. Авторы этой работы смогли описать природу спектра этой модели и найти ее зависимость от параметров модели.

Настоящая работа посвящена описанию асимптотик решений уравнения (1.1) при $x \rightarrow+\infty$ (теорема 1). При этом мы используем технику, разработанную в [4], [6]. Опираясь на полученные асимптотические разложения, мы описываем некоторые спектральные свойства уравнения (1.1) (теорема 2 и следствие 1).

Исходной для нас была работа Буслаева [5], посвященная уравнению (1.1) с линейным потенциалом $F(x)=F_{1} x, \quad F_{1}>0$, и периодическим потенциалом

$$
p(x)=V \sum_{n=-\infty}^{+\infty} \delta(x-n) .
$$

В этой работе был предложен метод исследования асимптотического поведения решений уравнения (1.1) при $x \rightarrow+\infty$. Впоследствии с помощью этого метода были получены асимптотики решений уравнения (1.1) для потенциалов $F(x)=F_{1} x$ и $p(x) \in L_{1, \text { loc }}(\mathbb{R})[4]$.

Опишем основную идею построений. Отметим сначала следующий факт. Заменим в уравнении (1.1) сумму $E+F(x)$ на $\mathcal{E}$ и забудем на некоторое время о том, что $\mathcal{E}$ зависит от $x$. В результате мы получим уравнение с периодическим потенциалом:

$$
-\psi^{\prime \prime}+p(x) \psi=\mathcal{E} \psi, \quad x \in \mathbb{R}_{+} .
$$


Далекие лакуны в спектре чисто периодического уравнения располагаются около точек $\mathcal{E}=(\pi l)^{2}$. Как известно, решения уравнения (1.2) ведут себя существенно различным образом, если $\mathcal{E}$ лежит в лакуне или на абсолютно непрерывном спектре соответствующего оператора. Поэтому можно ожидать, что точки поворота $\hat{n}_{l}$, удовлетворяющие уравнениям $E+F\left(\hat{n}_{l}\right)=(\pi l)^{2}, \quad l \in \mathbb{N}$, играют особую роль при изучении асимптотического поведения решений уравнения (1.1). Забегая вперед, отметим, что решения уравнения (1.1) ведут себя существенно по-разному в окрестности точек поворота и вне их. В соответствии с этим поиск асимптотик решений разбивается на несколько этапов. Во-первых, мы строим асимптотики решений уравнения (1.1) вне относительно малых окрестностей точек поворота $\hat{n}_{l}$. При этом возможность асимптотического описания решений основана на адиабатических соображениях. Во-вторых, мы строим асимптотики решений в окрестности точек поворота $\hat{n}_{l}$. Возможность построения эффективных асимптотик в данном случае опирается на малость лакун в спектре периодического уравнения. В-третьих, мы сшиваем полученные асимптотические разложения и тем самым получаем асимптотики решений при $x \rightarrow+\infty$.

Сформулируем основную для данной работы теорему.

ТЕОрема 1. Пусть потенциалы $p(x)$ и $F(x)$ удовлетворяют условиям (A1) $u$ (A2), соответственно. Пусть выполнена оценка

$$
s p_{l}=O\left(l^{1-\varepsilon}\right), \quad s p_{l}=\sup _{k \in[l-1, l]} \sup _{0 \leqslant x \leqslant 1}\left|\int_{0}^{x} p(t) e^{2 i \pi k t} d t\right|,
$$

где постоянная є взята из условия (А2).

Тогда прочзвольное решение $\psi$ уравнения (1.1) допускает при $x \rightarrow+\infty$ асимптотическое разложение вида

$$
\psi(x)=\frac{s_{l}}{\sqrt[4]{F(x)}} e^{i \Phi(x)}+\frac{t_{l}}{\sqrt[4]{F(x)}} e^{-i \Phi(x)}+O\left(\frac{\left\|\mathbf{s}_{l}\right\|}{\sqrt{F(x)}}\right), \quad x \in I_{l},
$$

где

$$
\Phi(x)=\int_{x_{0}}^{x} \sqrt{E+F(t)} d t, \quad \mathbf{s}_{l}=\left(\begin{array}{c}
s_{l} \\
t_{l}
\end{array}\right), \quad\left\|\mathbf{s}_{l}\right\|^{2}=s_{l}^{2}+t_{l}^{2},
$$

значение $x_{0} \in \mathbb{N}$ таково, что $E+F\left(x_{0}\right)>0$. Интервалы $I_{l}$ задаются условием

$$
I_{l}=\left(\tilde{n}_{l-1}, \tilde{n}_{l}\right) \backslash\left(\tilde{i}_{l-1} \cup \tilde{i}_{l}\right), \quad \tilde{i}_{l}=\left\{n:\left|n-\tilde{n}_{l}\right| \leqslant l^{2 \varepsilon / 3}\right\}, \quad l \in \mathbb{N} .
$$

Точки поворота $\tilde{n}_{l}$ задаются равенствами

$$
\begin{gathered}
\Delta\left(\tilde{n}_{l}\right)=\pi l, \quad \Delta(n)=k_{n}+\frac{o_{n}}{2 k_{n}}, \\
k_{n}=\sqrt{E+F(n)}, \quad o_{n}=\int_{0}^{1}(F(n+t)-F(n)) d t .
\end{gathered}
$$

Коэббициенты $s_{l}, t_{l}$ и $s_{l+1}, t_{l+1}$ на смежных интервалах $I_{l}$ u $I_{l+1}$ связаны преобразованием

$$
\mathbf{s}_{l+1}=W_{l} \mathbf{s}_{l},
$$


где $W_{l}$ допускает представление вида $W_{l}=e^{-i \Theta_{l} \sigma_{3}} S_{l} e^{i \Theta_{l} \sigma_{3}}$ nри

$$
\begin{aligned}
& \Theta_{l}=\sum_{k=x_{0}}^{n_{l}-1} \Delta(k), \quad \sigma_{1}=\left(\begin{array}{ll}
0 & 1 \\
1 & 0
\end{array}\right), \quad \sigma_{3}=\left(\begin{array}{cc}
1 & 0 \\
0 & -1
\end{array}\right), \\
& S_{l}=\left(\begin{array}{cc}
1+O(\varrho(l)) & \bar{r}(l) \kappa(l) l^{-1 / 2}+O(\varrho(l)) \\
r(l) \kappa(l) l^{-1 / 2}+O(\varrho(l)) & 1+O(\varrho(l))
\end{array}\right), \quad \sigma_{1} \overline{S_{l}} \sigma_{1}=S_{l}, \\
& \varrho(l)=l^{-1-\min (1, \varepsilon / 6)}+s p_{l} l^{-1}+s p_{l} l^{-2+\varepsilon}+\left(c p_{l}\right)^{2} l^{-2+\varepsilon} \ln l, \\
& r(l)=e^{i 3 \pi / 4} \frac{1}{\sqrt{2}} \int_{0}^{1} p(x) e^{2 i \pi l x} d x, \\
& c p_{l}=\sup _{k \in[l-1, l]}\left|\int_{0}^{1} p(t) e^{2 i \pi k t} d t\right|, \quad \kappa(l)=\left(F^{\prime}\left(\tilde{n}_{l}\right)\right)^{-1 / 2} .
\end{aligned}
$$

При этом выполнено равенство

$$
\int_{n_{l-1}}^{n_{l}}|\psi(x)|^{2} d x=\frac{2 \pi}{F^{\prime}\left(\tilde{n}_{l}\right)}\left\|\mathbf{s}_{l}\right\|^{2}(1+o(1)) .
$$

ЗАмечание 2. Отметим, что условие (1.3) выполнено всегда при $0<\varepsilon \leqslant 1$.

Из теоремы 1 можно вывести следующее утверждение.

ТЕОРема 2. Пусть выполнены предположения теоремы 1 и справедливы оценки

$$
\sum_{l=1}^{\infty}|r(l)| l^{\frac{\varepsilon-2}{2}}<\infty, \quad \sum_{l=1}^{\infty} \varrho(l)<\infty .
$$

Тогда спектр оператора $H$ чисто абсолютно непрерывен и заполняет всю вещественную осъ.

Сформулируем простейшее следствие из теоремы 2.

СледствиЕ 1. Пусть потенциал $F(x)$ удовлетворяет условию (А2) при некотором $\varepsilon \in(0,2)$. Пусть потенииал $p(x)$ удовлетворяет условию (A1) и является непрерывно дифберенцируемой функиией. Тогда спектр оператора $Н$ чисто абсолютно непрерывен и заполняет всю вещественную осъ.

ДоКАЗАТЕЛЬСТво. С помощью интегрирования по частям можно получить следующие оценки: $r(l)=O\left(l^{-1}\right), \quad s p_{l}=O\left(l^{-1}\right)$ и $c p_{l}=O\left(l^{-1}\right)$. Для завершения доказательства осталось воспользоваться теоремой 2.

Структура настоящей работы такова. Следующие пять разделов данной работы посвящены доказательству теоремы 1. При этом доказательство разбито на несколько этапов. В разделе 2 мы сводим уравнение (1.1) к дискретной системе, которая описывает связь между решениями уравнения (1.1) на соседних интервалах $[n, n+1]$ и $[n+1, n+2], n \in \mathbb{N}$. В разделе 3 мы находим адиабатические решения полученной системы вне относительно малых окрестностей точек поворота. В разделе 4 
мы находим решения системы в окрестности точек поворота. В разделе 5 мы описываем связь между полученными решениями дискретной системы. В разделе 6 мы возвращаемся от дискретной системы к исходному уравнению (1.1) и завершаем построение асимптотик решений уравнения (1.1). В разделе 7 мы доказываем теорему 2 .

\section{2. ДИСКРЕТНАЯ СИСТЕМА}

Преобразуем уравнение (1.1) к дискретной системе, которая описывает связь между решениями этого уравнения на интервалах $[n, n+1], n \in \mathbb{N}$.

Введем специальный базис решений исходного уравнения

$$
-\psi^{\prime \prime}-F(x) \psi+p(x) \psi=E \psi, \quad E \in \mathbb{R} .
$$

Пусть $\theta_{n}(x)$ и $\varphi_{n}(x)$ - такие решения уравнения $(2.1)$, что

$$
\begin{array}{ll}
\theta_{n}(n)=k_{n}^{-1 / 2}, & \varphi_{n}(n)=i k_{n}^{-1 / 2}, \\
\theta_{n}^{\prime}(n)=i k_{n}^{1 / 2}, & \varphi_{n}^{\prime}(n)=k_{n}^{1 / 2},
\end{array}
$$

При этом

$$
W\left(\theta_{n}(x), \varphi_{n}(x)\right)=\theta_{n}(x) \varphi_{n}^{\prime}(x)-\theta_{n}^{\prime}(x) \varphi_{n}(x)=2 .
$$

Отметим, что решения $\theta_{n}(x)$ и $\varphi_{n}(x)$ определены при достаточно больших значениях параметра $n$, более точно, при $E+F(n)>0$. Поскольку мы изучаем поведение решений на бесконечности, то всегда будем предполагать, что последнее условие выполнено.

На интервале $[n, n+1]$ произвольное решение уравнения $(2.1)$ может быть представлено в виде

$$
\psi(x)=a_{1}(n) \theta_{n}(x)+a_{2}(n) \varphi_{n}(x) .
$$

Так как решение $\psi(x)$ должно быть непрерывно дифференцируемо в точках $x=n$, то коэффициенты $a_{1}(n)$ и $a_{2}(n)$ должны удовлетворять следующему уравнению:

$$
\mathbf{a}(n+1)=T(n) \mathbf{a}(n), \quad \mathbf{a}(n)=\left(\begin{array}{c}
a_{1}(n) \\
a_{2}(n)
\end{array}\right)
$$

где матрица монодромии $T(n)$ задается равенством

$$
T(n)=\left(\begin{array}{ll}
\theta_{n+1}(n+1) & \varphi_{n+1}(n+1) \\
\theta_{n+1}^{\prime}(n+1) & \varphi_{n+1}^{\prime}(n+1)
\end{array}\right)^{-1}\left(\begin{array}{cc}
\theta_{n}(n+1) & \varphi_{n}(n+1) \\
\theta_{n}^{\prime}(n+1) & \varphi_{n}^{\prime}(n+1)
\end{array}\right) .
$$

Из тождества $(2.2)$ следует, что $\operatorname{det} T(n)=1$.

Матрица $T(n)$ при $E+F(n)>0$ удовлетворяет соотношению

$$
\sigma_{1} \overline{T(n)} \sigma_{1}=T(n) .
$$

Это следует из того факта, что $\overline{\varphi_{n}(x)}=-i \theta_{n}(x)$ при $E+F(n)>0$. 
Из формулы (2.6) следует, что если известно одно решение $\mathbf{a}^{(1)}(n)$ уравнения $(2.4)$, второе решение можно найти в виде

$$
\mathbf{a}^{(2)}(n)=\sigma_{1} \overline{\mathbf{a}^{(1)}(n)} \text {. }
$$

С учетом принятых обозначений произвольное решение $\psi$ уравнения $(2.1)$ может быть записано как

$$
\psi(x)=\left(\mathbf{a}(n), \mathbf{w}_{n}(x)\right)_{\mathrm{r}}, \quad \mathbf{w}_{n}(x)=\left(\begin{array}{c}
\theta_{n}(x) \\
\varphi_{n}(x)
\end{array}\right), \quad x \in[n, n+1] .
$$

Здесь и далее вещественное скалярное произведение отмечено индексом r, a комплексное скалярное произведение оставлено без индекса:

$$
(\mathbf{a}, \mathbf{b})_{\mathrm{r}}=a_{1} b_{1}+a_{2} b_{2}, \quad(\mathbf{a}, \mathbf{b})=a_{1} \bar{b}_{1}+a_{2} \bar{b}_{2}
$$

Из интегральных уравнений

$$
\begin{aligned}
& \theta_{n}(x)=\frac{1}{\sqrt{k_{n}}} e^{i k_{n}(x-n)}+\frac{1}{k_{n}} \int_{n}^{x} \sin \left\{k_{n}(x-t)\right\}(p(t)+F(n)-F(t)) \theta_{n}(t) d t, \\
& \varphi_{n}(x)=-\frac{1}{i \sqrt{k_{n}}} e^{-i k_{n}(x-n)}+\frac{1}{k_{n}} \int_{n}^{x} \sin \left\{k_{n}(x-t)\right\}(p(t)+F(n)-F(t)) \varphi_{n}(t) d t
\end{aligned}
$$

(величины $\Delta(n)$ и $k_{n}$ введены в (1.4)) можно получить асимптотику при $n \rightarrow \infty$ для матрицы $T(n)$ :

$$
T(n)=e^{i \Delta(n) \sigma_{3}} t(n),
$$

где

$$
t(n)=\left(\begin{array}{cc}
1+O\left(\frac{\omega(n)}{k_{n}^{2}}\right) & \frac{\bar{c}\left(k_{n}\right)}{2 k_{n}}+O\left(\frac{\omega(n)}{k_{n}^{2}}\right) \\
\frac{c\left(k_{n}\right)}{2 k_{n}}+O\left(\frac{\omega(n)}{k_{n}^{2}}\right) & 1+O\left(\frac{\omega(n)}{k_{n}^{2}}\right)
\end{array}\right) .
$$

Здесь

$$
c(k)=\int_{0}^{1} p(t) e^{2 i k t} d t, \quad \omega(n)=\left(1+k_{n}^{1-\varepsilon}\right)\left(k_{n}^{-\varepsilon}+\sup _{0 \leqslant x \leqslant 1}\left|\int_{0}^{x} p(t) e^{2 i k_{n} t} d t\right|\right) .
$$

\section{3. АДИАБАТИЧЕСКИЕ РЕШЕНИЯ}

В этом разделе мы рассмотрим асимптотики при $n \rightarrow \infty$ решений системы $(2.4)$ вне относительно малых окрестностей точек поворота.

Для больших $n$ система (2.4) может быть решена адиабатически. Это означает, что асимптотически решение может быть представлено в виде

$$
\mathbf{a}(n)=e^{i \Theta(n)} \mathbf{d}(n),
$$

где фаза $\Theta$ - вещественная функция и $\mathbf{d}-$ медленно меняющаяся функция. Мы можем найти такие решения всюду вне относительно малых окрестностей точек поворота. 
Чтобы построить адиабатические решения, подставим представление (3.1) в систему (2.4):

$$
e^{i \chi(n)} \mathbf{d}(n+1)=T(n) \mathbf{d}(n), \quad \chi(n)=\Theta(n+1)-\Theta(n) .
$$

Вектор-функцию $\mathbf{d}(n)$ будем искать в виде

$$
\mathbf{d}(n)=\mathbf{d}_{0}(n)+\mathbf{d}_{1}(n)+\mathbf{d}_{2}(n)
$$

При этом мы будем предполагать, что $\mathbf{d}_{1}=o\left(\mathbf{d}_{0}\right), \mathbf{d}_{2}=o\left(\mathbf{d}_{1}\right)$. Подставляя выражение (3.3) в систему (3.2) и приравнивая соответствующие коэффициенты, получим уравнения на $\mathbf{d}_{0}, \mathbf{d}_{1}$ и $\mathbf{d}_{2}$ :

$$
\begin{aligned}
\left(T(n)-e^{i \chi(n)}\right) \mathbf{d}_{0}(n) & =0, \\
\left(I-e^{-i \chi(n)} T(n)\right) \mathbf{d}_{1}(n) & =-\Delta \mathbf{d}_{0}(n), \quad \Delta \mathbf{d}_{0}(n) \stackrel{\text { def }}{=} \mathbf{d}_{0}(n+1)-\mathbf{d}_{0}(n), \\
\mathbf{d}_{2}(n+1)-e^{-i \chi(n)} T(n) \mathbf{d}_{2}(n) & =-\Delta \mathbf{d}_{1}(n), \quad \Delta \mathbf{d}_{1}(n) \stackrel{\text { def }}{=} \mathbf{d}_{1}(n+1)-\mathbf{d}_{1}(n) .
\end{aligned}
$$

Функция $\chi$ находится из условия разрешимости уравнения (3.4)

$$
\operatorname{det}\left(T(n)-e^{i \chi(n)}\right)=0
$$

Учитывая, что $\operatorname{det} T(n)=1$, последнее уравнение можно переписать в виде $\cos \chi(n)=$ $\operatorname{tr} T(n) / 2$. Отсюда

$$
\begin{gathered}
\cos \chi(n)=\cos \Delta(n)+O\left(C_{c \omega}(n)\right)+O\left(\frac{\omega(n) \sin \Delta(n)}{k_{n}^{2}}\right), \\
C_{c \omega}(n)=\frac{c^{2}\left(k_{n}\right)}{k_{n}^{2}}+\frac{\omega^{2}(n)}{k_{n}^{4}} \\
\chi(n)=\Delta(n)+O\left(\frac{C_{c \omega}(n)}{\sin \Delta(n)}\right)+O\left(\frac{\omega(n)}{k_{n}^{2}}\right) .
\end{gathered}
$$

Представление (3.7) справедливо вне окрестностей нулей функции $\sin \Delta(n)$. Из условия (А2) на потенциал $F(x)$ несложно вывести, что, начиная с некоторого $n_{0}$, $\Delta(n)$ - строго возрастающая функция. Поэтому, начиная с некоторого $l_{0} \in \mathbb{N}$, существует единственное решение $\tilde{n}_{l}$ уравнения $\Delta\left(\tilde{n}_{l}\right)=\pi l$ для каждого целого $l \geqslant l_{0}$. Обозначим через $n_{l}$ ближайшее целое число к $\tilde{n}_{l}$.

Дальнейшие построения в этом разделе будем проводить на интервалах

$$
I_{l}=\left(n_{l-1}, n_{l}\right) \backslash\left(\tilde{i}_{l-1} \cup \tilde{i}_{l}\right), \quad \tilde{i}_{l}=\left\{n:\left|n-n_{l}\right| \leqslant f(l) \stackrel{\text { def }}{=}\left[l^{2 \varepsilon / 3}\right]\right\} .
$$

Здесь $\left[l^{2 \varepsilon / 3}\right]$ - целая часть числа $l^{2 \varepsilon / 3}$. Длина интервалов $I_{l}$ имеет порядок $l^{\varepsilon}$.

Пусть $x_{0}-$ натуральное число такое, что $E+F\left(x_{0}\right)>0$. Учитывая $(3.7)$, из

3 Теоретическая и математическая физика, т. 146, № 3, 2006 г. 
уравнения (3.2) найдем

$$
\begin{aligned}
\Theta(n) \stackrel{\text { def }}{=} & \sum_{k=x_{0}}^{n-1} \Delta(k)+\sum_{k=\tilde{n}_{l-1}+f(l-1)}^{n-1}(\chi(k)-\Delta(k))= \\
= & \sum_{k=x_{0}}^{n-1} \Delta(k)+O\left(c p_{l}^{2} l^{-2+\varepsilon} \ln l\right)+O\left(s p_{l} l^{-1}\right)+ \\
& +O\left(s p_{l} l^{-2+\varepsilon}\right)+O\left(l^{-2}\right)+O\left(l^{-1-2 \varepsilon}\right), \quad n \in I_{l} .
\end{aligned}
$$

Здесь мы учли следующую оценку: $\sum_{n \in I_{l}}|\sin \Delta(n)|^{-1}=O\left(l^{\varepsilon} \ln l\right)$.

Решение уравнения (3.4) можно записать в виде

$$
\mathbf{d}_{0}(n) \stackrel{\text { def }}{=}\left(\begin{array}{c}
\alpha_{0}(n) \\
\beta_{0}(n)
\end{array}\right), \quad \beta_{0}(n)=\alpha_{0}(n) \alpha_{+}(n),
$$

где

$$
\begin{gathered}
\alpha_{+}(n)=\frac{t_{21}}{e^{i \chi}-t_{22}}=e^{-i \Delta(n)} \frac{c\left(k_{n}\right)}{4 i k_{n}} \frac{1}{\sin \Delta(n)}+O\left(\frac{1}{\sin \Delta(n)} l^{-1-\varepsilon}\right), \\
T(n)=\left(\begin{array}{ll}
t_{11} & t_{12} \\
t_{21} & t_{22}
\end{array}\right) .
\end{gathered}
$$

Мы использовали следующее предположение:

$$
s p_{l}=O\left(l^{1-\varepsilon}\right) .
$$

Введем в рассмотрение решение уравнения

$$
\left(T-e^{i \chi}\right)^{*} \eta=0
$$

где

$$
\eta=\left(\begin{array}{c}
1 \\
\bar{\tau}
\end{array}\right), \quad \tau=\frac{t_{12}}{e^{i \chi}-t_{22}}=e^{i \Delta(n)} \frac{\bar{c}\left(k_{n}\right)}{4 i k_{n}} \frac{1}{\sin \Delta(n)}+O\left(\frac{1}{\sin \Delta(n)} l^{-1-\varepsilon}\right)
$$

Для разрешимости уравнения (3.5) необходимо и достаточно, чтобы векторы $\eta(n)$ и $\Delta \mathbf{d}_{0}(n)$ были ортогональны:

$$
\left(\Delta \mathbf{d}_{0}(n), \eta(n)\right)=\Delta \alpha_{0}(n)+\tau(n) \Delta\left(\alpha_{+} \alpha_{0}\right)(n)=0,
$$

следовательно,

$$
\frac{\Delta \alpha_{0}(n)}{\alpha_{0}(n)}=g(n), \quad g(n) \stackrel{\text { def }}{=}-\frac{\tau(n) \Delta \alpha_{+}(n)}{1+\tau(n) \alpha_{+}(n+1)} .
$$

Отсюда найдем $\alpha_{0}$ как решение уравнения (3.10),

$$
\alpha_{0}(n)=\prod_{k=\tilde{n}_{l-1}+f(l-1)}^{n-1}(1+g(k)) .
$$


Справедливы следующие оценки:

$$
\begin{gathered}
g(k)=O\left(\frac{c p_{l}^{2}}{\sin ^{3} \Delta(n)} l^{-2-\varepsilon}+\frac{c p_{l}}{\sin ^{2} \Delta(n)} l^{-2-\varepsilon}\right), \\
\alpha_{0}(n)=1+O\left(c p_{l}^{2} l^{-2+2 \varepsilon / 3}+c p_{l} l^{-2+\varepsilon / 3}\right), \\
\beta_{0}(n)=e^{-i \Delta(n)} \frac{c\left(k_{n}\right)}{4 i k_{n}} \frac{1}{\sin \Delta(n)}+O\left(c p_{l}^{2} l^{-3+\varepsilon}\right), \\
\Delta \alpha_{0}(n)=O(g(n))=O\left(\frac{c p_{l}^{2}}{\sin ^{3} \Delta(n)} l^{-2-\varepsilon}+\frac{c p_{l}}{\sin ^{2} \Delta(n)} l^{-2-\varepsilon}\right), \\
\Delta \beta_{0}(n)=O\left(\frac{c p_{l}}{\sin ^{2} \Delta(n)} l^{-1-\varepsilon}+\frac{1}{\sin \Delta(n)} l^{-1-\varepsilon}\right) .
\end{gathered}
$$

В силу равенства (3.10) уравнение (3.5) разрешимо. Будем искать его решение в виде $\mathbf{d}_{1}(n)=\left(\begin{array}{c}0 \\ \beta_{1}(n)\end{array}\right)$. Подставляя это выражение в $(3.5)$, получим

$$
\begin{aligned}
\beta_{1}(n)=e^{i \chi(n)} \frac{\Delta \beta_{0}(n)}{t_{22}-e^{i \chi(n)}} & =O\left(\frac{c p_{l}}{\sin ^{3} \Delta(n)} l^{-1-\varepsilon}+\frac{1}{\sin ^{2} \Delta(n)} l^{-1-\varepsilon}\right), \\
\Delta \beta_{1}(n) & =O\left(\frac{c p_{l}}{\sin ^{4} \Delta(n)} l^{-1-2 \varepsilon}+\frac{1}{\sin ^{3} \Delta(n)} l^{-1-2 \varepsilon}\right) .
\end{aligned}
$$

Рассмотрим на интервале $I_{l}$ матричное уравнение

$$
V(n+1)=e^{-i \chi(n)} T(n) V(n), \quad V\left(\tilde{n}_{l-1}+f(l-1)\right)=I .
$$

Оценим его решения: $\|V(n)\| \leqslant\left(1+O\left(l^{-1} c p_{l}\right)+O\left(l^{-2 \varepsilon}\right)\right)^{l^{\varepsilon}}$. В силу (3.9) справедлива оценка $\|V(n)\|=O(1)$.

Для того чтобы оценить $\mathbf{d}_{2}(n)$, воспользуемся следующими оценками:

$$
\sum_{n \in I_{l}} \frac{1}{\left|\sin ^{p} \Delta(n)\right|}=O\left(l^{\varepsilon(p+2) / 3}\right), \quad p=2,3, \ldots
$$

Используя (3.12), найдем $\mathbf{d}_{2}(n)$ в виде

$$
\mathbf{d}_{2}(n)=-V(n) \sum_{k=\tilde{n}_{l-1}+f(l-1)}^{n} V^{-1}(k+1) \Delta \mathbf{d}_{1}(k)=O\left(c p_{l} l^{-1}+l^{-1-\varepsilon / 3}\right) .
$$

Собирая полученные оценки вместе и подставляя их в (3.3), получим следующее утверждение (ради удобства мы переопределили фазу $\Theta(n)$ и вынесли поправочные слагаемые из-под экспоненты в (3.13)).

Лемма 1. Пусть выполнена оценка (3.9). Тогда на интервалах $I_{l}$ существует решение $\mathbf{a}(n)$ уравнения (2.4) со следуюшим поведением при $n \rightarrow \infty$ :

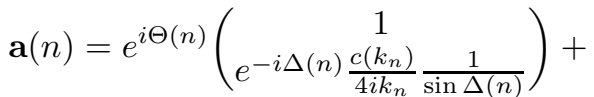

$$
\begin{aligned}
& +O\left(c p_{l}^{2} l^{-2+\varepsilon} \ln l\right)+O\left(s p_{l} l^{-1}\right)+O\left(s p_{l} l^{-2+\varepsilon}\right)+O\left(l^{-2}\right)+O\left(l^{-1-\varepsilon / 3}\right),
\end{aligned}
$$


где

$$
\Theta(n)=\sum_{k=x_{0}}^{n-1} \Delta(k)
$$

ЗАмечАниЕ 3 . На каждом интервале $I_{l}$ решение $\mathbf{a}(n)$ системы $(2.4)$ строится независимым образом. При этом на интервалах $\tilde{i}_{l}$ невозможно определить функцию $\mathbf{a}(n)$ так, чтобы она была решением системы (2.4) при всех $n$. Асимптотика (3.13) понимается в обычном смысле, но с одной оговоркой: она имеет силу только для $n \in \bigcup_{l \geqslant 0} I_{l}$ (для остальных $n$ функция $\mathbf{a}(n)$ не определена).

\section{4. ТОЧКИ ПОВОРОТА}

Этот раздел посвящен построению асимптотик решений при $n \rightarrow \infty$ уравнения (2.4) в относительно малых окрестностях точек поворота.

Перепишем систему (2.4) в виде

$$
\mathbf{a}(n+1)=e^{i \Delta(n) \sigma_{3}} t(n) \mathbf{a}(n) .
$$

Точки $\tilde{n}_{l}$ не являются целыми, поэтому вновь введем целое число $n_{l}$, ближайшее к $\tilde{n}_{l}$. Пусть вместе с этим $\widetilde{m}=n-\tilde{n}_{l}, m=n-n_{l}$. Рассмотрим уравнение (4.1) на интервалах $i_{l}$, более широких, чем $\tilde{i}_{l}$ :

$$
i_{l}=\left\{n:\left|n-n_{l}\right| \leqslant 2 f(l)\right\}, \quad \Delta\left(\tilde{n}_{l}\right)=\pi l .
$$

Основная идея решения уравнения (4.1) состоит в замене функции $\Delta(n)$ и матрицы $t(n)$ первыми членами рядов Тейлора в окрестности точки $\tilde{n}_{l}$. Используя представление (2.11), получим

$$
\begin{gathered}
\Delta(n)=\pi l+\frac{F^{\prime}\left(\tilde{n}_{l}\right)}{2 \pi l} \widetilde{m}+O\left(l^{-1-2 \varepsilon / 3}\right), \\
t(n)=I+\frac{1}{2 \pi l}\left(\begin{array}{cc}
0 & \bar{s}_{0}(l) \\
s_{0}(l) & 0
\end{array}\right)+\frac{F^{\prime}\left(\tilde{n}_{l}\right)}{2 \pi^{2} l^{2}}\left(\tilde{m}-\frac{1}{2}\right)\left(\begin{array}{cc}
0 & -i \bar{s}_{1}(l) \\
i s_{1}(l) & 0
\end{array}\right)+ \\
+O\left(s_{2}(l) l^{-1-2 \varepsilon / 3}\right)+O\left(l^{-2-2 \varepsilon / 3}\right)+O\left(l^{-1-\varepsilon}\right),
\end{gathered}
$$

где

$$
s_{0}(l)=c(\pi l)=\int_{0}^{1} p(t) e^{2 i \pi l t} d t, \quad s_{1}(l)=\int_{0}^{1} t p(t) e^{2 i \pi l t} d t, \quad s_{2}(l)=\int_{0}^{1} t^{2} p(t) e^{2 i \pi l t} d t .
$$

Введем в рассмотрение параметры

$$
\begin{gathered}
\delta_{0}=n_{l}-\tilde{n}_{l}, \quad \delta_{1}=n_{l}-\tilde{n}_{l}-\frac{1}{2}, \quad\left|\delta_{0}\right| \leqslant \frac{1}{2}, \quad\left|\delta_{1}\right| \leqslant 1, \\
\gamma_{0}^{2}=\frac{1}{2 \pi l}, \quad \gamma_{1}^{2}=\frac{F^{\prime}\left(\tilde{n}_{l}\right)}{2 \pi l}=O\left(l^{-\varepsilon}\right), \quad \gamma_{2}=\gamma_{0}^{2} \gamma_{1}^{-1}=O\left(l^{-1+\varepsilon / 2}\right) .
\end{gathered}
$$

Используя их, перепишем разложения для $\Delta(n)$ и $t(n)$ в виде

$$
\begin{gathered}
\Delta(n)=\pi l+\gamma_{1}^{2}\left(m+\delta_{0}\right)+O\left(l^{-1-2 \varepsilon / 3}\right), \\
t(n)=I+\gamma_{0}^{2} S_{0}(l)+2 \gamma_{0}^{2} \gamma_{1}^{2}\left(m+\delta_{1}\right) S_{1}(l)+O\left(s_{2}(l) l^{-1-2 \varepsilon / 3}\right)+O\left(l^{-2-2 \varepsilon / 3}\right)+O\left(l^{-1-\varepsilon}\right) .
\end{gathered}
$$



Здесь

$$
S_{0}(l)=\left(\begin{array}{cc}
0 & \bar{s}_{0}(l) \\
s_{0}(l) & 0
\end{array}\right), \quad S_{1}(l)=\left(\begin{array}{cc}
0 & -i \bar{s}_{1}(l) \\
i s_{1}(l) & 0
\end{array}\right)
$$

Сделаем в системе (4.1) подстановку

$$
\mathbf{a}(n)=\exp \left(i \sum_{k=n_{l}}^{n-1} \Delta(k) \sigma_{3}\right) \mathbf{g}\left(y+\delta_{1} \gamma_{1}\right), \quad y=\gamma_{1} m .
$$

В результате получим уравнение для $\mathbf{g}$ :

$$
\mathbf{g}\left(z+\gamma_{1}\right)=M_{1}(z) \mathbf{g}(z)
$$

где $z=y+\delta_{1} \gamma_{1}$,

$$
\begin{gathered}
M_{1}(z)=I+\gamma_{0}^{2} S_{0}(l, z)+2 \gamma_{0}^{2} \gamma_{1} z S_{1}(l, z)+O\left(d_{l}\right), \quad S_{p}(l, z)=S_{p}(l) e^{i z^{2} \sigma_{3}}, \quad p=0,1, \\
O\left(d_{l}\right)=O\left(c_{l} l^{-2}\right)+O\left(s_{1}(l) l^{-2-\varepsilon / 3}\right)+O\left(s_{2}(l) l^{-1-2 \varepsilon / 3}\right)+O\left(l^{-2-2 \varepsilon / 3}\right)+O\left(l^{-1-\varepsilon}\right) .
\end{gathered}
$$

Будем искать решение уравнения (4.5) в виде

$$
\mathbf{g}(z)=\mathbf{g}_{0}(z)+\gamma_{2} \mathbf{g}_{1}(z)+\gamma_{2}^{2} \mathbf{g}_{2}(z)+\mathbf{u}(z) .
$$

Раскладывая $\mathbf{g}\left(z+\gamma_{1}\right)$ в ряд Тейлора и приравнивая коэффициенты при одинаковых степенях $\gamma$ (при этом удобно считать, что $\gamma_{0}, \gamma_{1}$ и $\gamma_{2}$ одного порядка), получим уравнения на $\mathbf{g}_{0}, \mathbf{g}_{1}$ и $\mathbf{g}_{2}$

$$
\begin{aligned}
\gamma: & \mathbf{g}_{0}^{\prime}(z)=0 \\
\gamma^{2}: & \mathbf{g}_{1}^{\prime}(z)=S_{0}(l, z) \mathbf{g}_{0}(z), \\
\gamma^{3}: & \mathbf{g}_{2}^{\prime}(z)=S_{0}(l, z) \mathbf{g}_{1}(z)-\frac{1}{2} \gamma_{0}^{-2} \gamma_{1}^{2} \mathbf{g}_{1}^{\prime \prime}(z)+2 \gamma_{0}^{-2} \gamma_{1}^{2} z S_{1}(l, z) \mathbf{g}_{0}(z) .
\end{aligned}
$$

Введем обозначения

$$
R_{1}(z)=\int_{-\infty}^{z} e^{i t^{2}} d t, \quad R_{2}(z)=\int_{-f \gamma_{1}}^{z} e^{-i t^{2}} d t \int_{-\infty}^{t} e^{i s^{2}} d s, \quad R_{3}(z)=\int_{-f \gamma_{1}}^{z} t e^{i t^{2}} d t .
$$

Решая уравнения на $\mathbf{g}_{0}, \mathbf{g}_{1}$ и $\mathbf{g}_{2}$, получим

$$
\begin{aligned}
\mathbf{g}_{0}(z)= & \hat{\mathbf{g}}_{0}-\text { постоянный вектор, } \\
\mathbf{g}_{1}(z)= & S_{0}(l)\left(\begin{array}{cc}
R_{1}(z) & 0 \\
0 & \bar{R}_{1}(z)
\end{array}\right) \hat{\mathbf{g}}_{0}, \\
\mathbf{g}_{2}(z)= & \left(S_{0}(l)\right)^{2}\left(\begin{array}{cc}
R_{2}(z) & 0 \\
0 & \bar{R}_{2}(z)
\end{array}\right) \hat{\mathbf{g}}_{0}- \\
& -\frac{1}{2} \gamma_{0}^{-2} \gamma_{1}^{2} S_{0}(l) e^{i z^{2} \sigma_{3}} \hat{\mathbf{g}}_{0}+2 \gamma_{0}^{-2} \gamma_{1}^{2} S_{1}(l)\left(\begin{array}{cc}
R_{3}(z) & 0 \\
0 & \bar{R}_{3}(z)
\end{array}\right) \hat{\mathbf{g}}_{0} .
\end{aligned}
$$


Теперь необходимо подставить выражения (4.7)-(4.9) в систему (4.5), откуда получим уравнение на $\mathbf{u}(z)$ :

$$
\mathbf{u}\left(z+\gamma_{1}\right)-M_{1}(z) \mathbf{u}(z)=O\left(d_{l}\left(1+s_{1}(l) l^{-1+\varepsilon / 3}\right)\right)+O\left(l^{-1-5 \varepsilon / 6}\right) .
$$

Для того чтобы оценить решение и системы (4.10), введем матричное решение $U(z)$ системы

$$
U\left(z+\gamma_{1}\right)=M_{1}(z) U(z), \quad U(0)=I, \quad|z| \leqslant \gamma_{1} f
$$

Из представления (2.11) и оценки (3.9) следует, что

$$
\|U(z)\| \leqslant \max _{|z| \leqslant \gamma_{1} f}\left\|M_{1}(z)\right\|^{f}=\max _{n \in i_{l}}\|t(n)\|^{f} \leqslant C_{5},
$$

где постоянная $C_{5}$ не зависит от номера интервала $i_{l}$. Из $(3.9)$ следует, что выполнена оценка

$$
\left|s_{1}(l)\right|=O\left(l^{1-\varepsilon}\right) .
$$

Отсюда

$$
\begin{gathered}
U^{-1}\left(z+\gamma_{1}\right) \mathbf{u}\left(z+\gamma_{1}\right)-U^{-1}(z) \mathbf{u}(z)=O\left(d_{l}\right)+O\left(l^{-1-5 \varepsilon / 6}\right), \\
\mathbf{u}(z)=U(z) \sum_{z^{\prime}=0}^{z-\gamma_{1}}\left(O\left(d_{l}\right)+O\left(l^{-1-5 \varepsilon / 6}\right)\right), \\
\|\mathbf{u}(z)\| \leqslant\|U(z)\|\left(O\left(d_{l} f\right)+O\left(l^{-1-5 \varepsilon / 6} f\right)\right) \leqslant O\left(l^{-1-\min (1, \varepsilon / 6)}\right)+O\left(s_{2}(l) l^{-1}\right) .
\end{gathered}
$$

Подставляя выражения (4.7)-(4.9) и оценку (4.12) в формулу (4.6), получим следующее утверждение.

Лемма 2. Пусть выполнена оценка (4.11). Тогда на интервале $|z| \leqslant 2 \gamma_{1} f$ существует решение уравнения (4.5) со следующим асимптотическим поведением при $l \rightarrow \infty$ :

$$
\mathbf{g}(z)=\mathbf{g}_{0}(z)+\mathbf{g}_{1}(z)+\mathbf{g}_{2}(z)+O\left(l^{-1-\min (1, \varepsilon / 6)}\right)+O\left(s_{2}(l) l^{-1}\right),
$$

где вектор-функиии $\mathbf{g}_{0}(z), \mathbf{g}_{1}(z)$ и $\mathbf{g}_{2}(z)$ задаются равенствами (4.7)-(4.9).

Используя представление (4.4) и лемму 2, найдем асимптотики решений уравнения (4.1) на границе интервала $\tilde{i}_{l}$ :

$$
\begin{aligned}
\mathbf{g}(z)= & {\left[I+\gamma_{2} S_{0}\left(\begin{array}{cc}
R_{1}(z) & 0 \\
0 & \bar{R}_{1}(z)
\end{array}\right)\right] \hat{\mathbf{g}}_{0}+} \\
& +O\left(l^{-1-\min (1, \varepsilon / 6)}\right)+O\left(s_{0}(l) l^{-1}\right)+O\left(s_{2}(l) l^{-1}\right), \\
\mathbf{g}\left(y+\delta_{1} \gamma_{1}\right)= & {\left[I+\gamma_{2} S_{0}\left(\begin{array}{cc}
R_{1}(y) & 0 \\
0 & \bar{R}_{1}(y)
\end{array}\right)\right] \hat{\mathbf{g}}_{0}+} \\
& +O\left(l^{-1-\min (1, \varepsilon / 6)}\right)+O\left(s_{0}(l) l^{-1}\right)+O\left(s_{2}(l) l^{-1}\right) .
\end{aligned}
$$


При этом мы использовали следующие оценки:

$$
\begin{array}{cc}
R_{2}\left(-f \gamma_{1}\right)=0, \quad R_{2}\left(f \gamma_{1}\right)=\frac{\pi}{2}+o(1), & R_{3}\left( \pm f \gamma_{1}\right)=0, \\
R_{p}\left(y+\delta_{1} \gamma_{1}\right)=R_{p}(y)+O\left(\gamma_{1} R_{p}^{\prime}(y)\right), & p=1,2,3 .
\end{array}
$$

Таким образом, справедлива следующая лемма.

ЛЕмма 3. Пусть выполнена оценка (4.11). Тогда на интервалах $i_{l}$ существует решение уравнения (4.1) со следующим асимптотическим поведением при $l \rightarrow \infty$ на гранище интервала $\tilde{i}_{l}\left(\right.$ рри $\left.n=n_{l} \pm f(l)\right)$ :

$$
\mathbf{a}(n)=\exp \left(i \sum_{k=n_{l}}^{n-1} \Delta(k) \sigma_{3}\right) \mathbf{h}\left(\gamma_{1}\left(n-n_{l}\right)\right),
$$

гдe

$$
\mathbf{h}(y)=\left[I+\gamma_{2} S_{0}\left(\begin{array}{cc}
R_{1}(y) & 0 \\
0 & \bar{R}_{1}(y)
\end{array}\right)\right] \hat{\mathbf{g}}_{0}+O\left(l^{-1-\min (1, \varepsilon / 6)}\right)+O\left(s_{0}(l) l^{-1}\right)+O\left(s_{2}(l) l^{-1}\right) .
$$

\section{5. ПЕРЕХОД ЧЕРЕЗ ТОЧКИ ПОВОРОТА}

Итак, мы нашли поведение решений уравнения (2.4) на интервалах $I_{l}$ и $i_{l}$, определенных формулами (3.8) и (4.2), соответственно. Нетрудно заметить, что интервал $i_{l}$ пересекается с двумя интервалами $I_{l}$ и $I_{l+1}$, поэтому полученные решения можно сшить.

Поведение решения уравнения (2.4) на интервале $I_{l}$ описывается выражением

$$
\begin{aligned}
\mathbf{a}_{l}^{(1)}(n)=e^{i \sum_{k=x_{0}}^{n-1} \Delta(k)}\left(\begin{array}{c}
1 \\
\frac{e^{-i \Delta(n)} c\left(k_{n}\right)}{4 i k_{n} \sin \Delta(n)}
\end{array}\right)+O\left(c p_{l}^{2} l^{-2+\varepsilon} \ln l\right)+ \\
\quad+O\left(s p_{l} l^{-1}\right)+O\left(s p_{l} l^{-2+\varepsilon}\right)+O\left(l^{-2}\right)+O\left(l^{-1-\varepsilon / 3}\right) .
\end{aligned}
$$

Пусть $\mathbf{a}_{l}^{(2)}$ - решение, заданное равенством $(2.7), \mathbf{a}_{l}^{(2)}(n)=\sigma_{1} \overline{\mathbf{a}_{l}^{(1)}(n)}$.

Введем обозначение

$$
\Theta_{l}=\sum_{k=x_{0}}^{n_{l}-1} \Delta(k)
$$

С помощью представления (5.1) найдем поведение решений $\mathbf{a}_{l}^{(1)}$ и $\mathbf{a}_{l+1}^{(1)}$ на границе интервала $\tilde{i}_{l}$ :

на левой границе $\left(n=n_{l}-f(l)\right)$ имеем

$$
\mathbf{a}_{l}^{(1)}(n)=e^{i \Theta_{l}} \exp \left(-i \sum_{k=n_{l}-f}^{n_{l}-1} \Delta(k)\right)\left(\begin{array}{c}
1 \\
-\frac{s_{0}(l)}{4 i \pi l f \gamma_{1}^{2}}
\end{array}\right)+O(\varrho(l))
$$

на правой границе $\left(n=n_{l}+f(l)\right)$ имеем

$$
\mathbf{a}_{l+1}^{(1)}(n)=e^{i \Theta_{l}} \exp \left(i \sum_{k=n_{l}}^{n_{l}+f-1} \Delta(k)\right)\left(\begin{array}{c}
1 \\
\frac{s_{0}(l)}{4 i \pi l f \gamma_{1}^{2}}
\end{array}\right)+O(\varrho(l))
$$

(обозначение $\varrho(l)$ введено после формулы (1.5)). 
В разделе 4 мы построили решение уравнения (2.4) на интервале $i_{l}$ вида (4.6). Обозначим это решение через $A_{1}$ при $\hat{\mathbf{g}}_{0}=\left(\begin{array}{l}1 \\ 0\end{array}\right)$. Введем в рассмотрение второе решение $A_{2}(n)=\sigma_{1} \overline{A_{1}(n)}$ в соответствии с формулой (2.7). Асимптотическое поведение решений $A_{1}$ и $A_{2}$ на границе интервала $\tilde{i}_{l}$ описывается леммой 3 :

на левой границе $\left(n=n_{l}-f(l)\right)$

$$
A_{1}(n)=\exp \left(-i \sum_{k=n_{l}-f}^{n_{l}-1} \Delta(k) \sigma_{3}\right)\left(\begin{array}{c}
1 \\
\gamma_{2} s_{0}(l) R_{1}\left(-f \gamma_{1}\right)
\end{array}\right)+O(\varrho(l))
$$

на правой границе $\left(n=n_{l}+f(l)\right)$

$$
A_{1}(n)=\exp \left(i \sum_{k=n_{l}}^{n_{l}+f-1} \Delta(k) \sigma_{3}\right)\left(\begin{array}{c}
1 \\
\gamma_{2} s_{0}(l) R_{1}\left(f \gamma_{1}\right)
\end{array}\right)+O(\varrho(l))
$$

Используя асимптотические формулы

$$
\begin{aligned}
R_{1}\left(-f \gamma_{1}\right) & =-\frac{1}{2 i f \gamma_{1}} e^{i f^{2} \gamma_{1}^{2}}+O\left(f^{-3} \gamma_{1}^{-3}\right) \\
R_{1}\left(f \gamma_{1}\right) & =R_{1}(+\infty)+\frac{1}{2 i f \gamma_{1}} e^{i f^{2} \gamma_{1}^{2}}+O\left(f^{-3} \gamma_{1}^{-3}\right)
\end{aligned}
$$

перепишем разложения (5.5) и (5.6):

на левой границе $\left(n=n_{l}-f(l)\right)$

$$
A_{1}(n)=\exp \left(-i \sum_{k=n_{l}-f}^{n_{l}-1} \Delta(k) \sigma_{3}\right)\left(\begin{array}{c}
1 \\
-\frac{s_{0}(l)}{4 i \pi l f \gamma_{1}^{2}} e^{i f^{2} \gamma_{1}^{2}}
\end{array}\right)+O(\varrho(l))
$$

на правой границе $\left(n=n_{l}+f(l)\right)$

$$
A_{1}(n)=\exp \left(i \sum_{k=n_{l}}^{n_{l}+f-1} \Delta(k) \sigma_{3}\right)\left[\left(\begin{array}{c}
1 \\
\frac{s_{0}(l)}{4 i \pi l f \gamma_{1}^{2}} e^{i f^{2} \gamma_{1}^{2}}
\end{array}\right)+\left(\begin{array}{c}
0 \\
\gamma_{2} s_{0}(l) R_{1}(+\infty)
\end{array}\right)\right]+O(\varrho(l))
$$

На интервалах $i_{l}$ справедливо равенство

$$
\Delta(n)=\pi l+\gamma_{1}^{2}\left(m+\delta_{0}\right)+O\left(l^{-1-2 \varepsilon / 3}\right), \quad m=n-n_{l},
$$

отсюда

$$
\begin{aligned}
& 2 \sum_{k=n_{l}-f}^{n_{l}-1} \Delta(k)=2 \pi l f-\gamma_{1}^{2} f(f-1)+2 \delta_{0} f \gamma_{1}^{2}+O\left(l^{-1}\right), \\
& 2 \sum_{k=n_{l}}^{n_{l}+f-1} \Delta(k)=2 \pi l f+\gamma_{1}^{2} f(f-1)+2 \delta_{0} f \gamma_{1}^{2}+O\left(l^{-1}\right) .
\end{aligned}
$$

Подставляя полученные выражения в (5.7) и (5.8), получим 
на левой границе $\left(n=n_{l}-f(l)\right)$

$$
A_{1}(n)=\exp \left(-i \sum_{k=n_{l}-f}^{n_{l}-1} \Delta(k)\right)\left(\begin{array}{c}
1 \\
-\frac{s_{0}(l)}{4 i \pi l f \gamma_{1}^{2}}
\end{array}\right)+O(\varrho(l)),
$$

на правой границе $\left(n=n_{l}+f(l)\right)$

$$
\begin{aligned}
A_{1}(n)=\exp & \left(i \sum_{k=n_{l}}^{n_{l}+f-1} \Delta(k)\right)\left(\begin{array}{c}
1 \\
\frac{s_{0}(l)}{4 i \pi l f \gamma_{1}^{2}}
\end{array}\right)+ \\
& +\exp \left(-i \sum_{k=n_{l}}^{n_{l}+f-1} \Delta(k)\right)\left(\begin{array}{c}
0 \\
\gamma_{2} s_{0}(l) R_{1}(+\infty)
\end{array}\right)+O(\varrho(l)) .
\end{aligned}
$$

Используя асимптотики (5.3), (5.9) и (5.4), (5.10), найдем связь между решениями $\mathbf{a}_{l}, \mathbf{a}_{l+1}$ и $A_{1}$ :

$$
A_{1}(n)=e_{1} \mathbf{a}_{l}^{(1)}(n)+e_{2} \mathbf{a}_{l}^{(2)}(n)=g_{1} \mathbf{a}_{l+1}^{(1)}(n)+g_{2} \mathbf{a}_{l+1}^{(2)}(n)
$$

где

$$
\begin{aligned}
& e_{1}=e^{-i \Theta_{l}}(1+O(\varrho(l))), \quad e_{2}=O(\varrho(l)), \\
& g_{1}=e^{-i \Theta_{l}}(1+O(\varrho(l))), \quad g_{2}=e^{i \Theta_{l}}\left(\gamma_{2} s_{0}(l) R_{1}(+\infty)+O(\varrho(l))\right) \text {. }
\end{aligned}
$$

Учитывая соотношения $A_{2}(n)=\sigma_{1} \overline{A_{1}(n)}$ и $\mathbf{a}_{l}^{(2)}(n)=\sigma_{1} \overline{\mathbf{a}_{l}^{(1)}(n)}$, получим

$$
A_{2}(n)=\bar{e}_{2} \mathbf{a}_{l}^{(1)}(n)+\bar{e}_{1} \mathbf{a}_{l}^{(2)}(n)=\bar{g}_{2} \mathbf{a}_{l+1}^{(1)}(n)+\bar{g}_{1} \mathbf{a}_{l+1}^{(2)}(n)
$$

Из соотношений (5.11) и (5.12) следует, что

$$
\left(\begin{array}{c}
\mathbf{a}_{l+1}^{(1)} \\
\mathbf{a}_{l+1}^{(2)}
\end{array}\right)=N_{l}\left(\begin{array}{c}
\mathbf{a}_{l}^{(1)} \\
\mathbf{a}_{l}^{(2)}
\end{array}\right)
$$

где $N_{l}=e^{i \Theta_{l} \sigma_{3}} M_{l} e^{-i \Theta_{l} \sigma_{3}}$,

$$
M_{l}=\left(\begin{array}{cc}
1+O(\varrho(l)) & -R_{1}(\infty) \gamma_{2} s_{0}(l)+O(\varrho(l)) \\
-\overline{R_{1}(\infty)} \gamma_{2} \bar{s}_{0}(l)+O(\varrho(l)) & 1+O(\varrho(l))
\end{array}\right) .
$$

Из соотношения (2.7) следует, что матрица $M_{l}$ удовлетворяет тождеству

$$
\sigma_{1} \overline{M_{l}} \sigma_{1}=M_{l}
$$

Суть равенства (5.13) состоит в следующем: решения $\mathbf{a}_{l}^{(1)}$ и $\mathbf{a}_{l}^{(2)}$ уравнения (2.4) после продолжения через интервал $i_{l}$ переходят в линейную комбинацию решений $\mathbf{a}_{l+1}^{(1)}$ и $\mathbf{a}_{l+1}^{(2)}$, которая задается равенством (5.13). Отметим, что решения $\mathbf{a}_{l}^{(1)}(n)$ и $\mathbf{a}_{l}^{(2)}(n)$ определены при всех значениях аргумента $n$, однако их асимптотическое поведение определено только на интервалах $I_{l}$. 


\section{6. АСИМПТОТИКИ РЕШЕНИЙ УРАВНЕНИЯ ШРЕДИНГЕРА}

Этот раздел посвящен описанию асимптотик решений исходного уравнения (2.1) на интервалах $I_{l}$ и формулировке условий связи между этими асимптотиками на соседних интервалах $I_{l}$ и $I_{l+1}$.

На интервале $I_{l}$ произвольное решение уравнения (2.4) может быть представлено в виде

$$
\mathbf{a}(n)=\hat{s}_{l} \mathbf{a}_{l}^{(1)}(n)+\hat{t}_{l} \mathbf{a}_{l}^{(2)}(n), \quad n \in I_{l},
$$

где $\hat{s}_{l}$ и $\hat{t}_{l}-$ константы.

Введем вектор $\hat{\mathbf{s}}_{l}=\left(\begin{array}{l}\hat{s}_{l} \\ t_{l}\end{array}\right)$. Используя (5.13), легко найти связь между $\hat{\mathbf{s}}_{l+1}$ и $\hat{\mathbf{s}}_{l}$ :

$$
\hat{\mathbf{s}}_{l+1}=\widehat{W}_{l} \hat{\mathbf{s}}_{l}, \quad \widehat{W}_{l}=\left(N_{l}^{t}\right)^{-1} .
$$

Согласно соотношению $(2.8)$ на интервале $[n, n+1] \subset I_{l}$ произвольное решение $\psi$ уравнения (2.1) может быть записано в виде

$$
\psi(x)=\left(\hat{s}_{l} \mathbf{a}_{l}^{(1)}(n)+\hat{t}_{l} \mathbf{a}_{l}^{(2)}(n),\left(\begin{array}{c}
\theta_{n}(x) \\
\varphi_{n}(x)
\end{array}\right)\right)_{\mathrm{r}} .
$$

Используя лемму 1 и интегральные уравнения (2.9), (2.10), получим

$$
\begin{aligned}
\psi(x) & =\left(\left(\hat{s}_{l} e^{i \Theta(n)}\left(\begin{array}{l}
1 \\
0
\end{array}\right)+\hat{t}_{l} e^{-i \Theta(n)}\left(\begin{array}{l}
0 \\
1
\end{array}\right)+O\left(l^{-1} \ln l\right)\right),\left(\begin{array}{c}
\theta_{n}(x) \\
\varphi_{n}(x)
\end{array}\right)\right)_{\mathrm{r}}= \\
& =\hat{s}_{l} e^{i \Theta(n)} \theta_{n}(x)+\hat{t}_{l} e^{-i \Theta(n)} \varphi_{n}(x)+k_{n}^{-1} O\left(\left\|\hat{\mathbf{s}}_{l}\right\| l^{-1} \ln l\right)= \\
& =\frac{\hat{s}_{l}}{\sqrt{k_{n}}} e^{i \Theta(n)} e^{i k_{n}(x-n)}+\frac{i \hat{t}_{l}}{\sqrt{k_{n}}} e^{-i \Theta(n)} e^{-i k_{n}(x-n)}+O\left(\left\|\hat{\mathbf{s}}_{l}\right\| l^{-1}\right) .
\end{aligned}
$$

Справедлива цепочка равенств

$$
\begin{aligned}
\int_{x_{0}}^{x} \sqrt{E+F(t)} d t=\int_{x_{0}}^{x} k_{t} d t=\sum_{p=x_{0}}^{n-1} \int_{p}^{p+1} k_{t} d t+\int_{n}^{x} k_{t} d t= \\
=\sum_{p=x_{0}}^{n-1} \int_{p}^{p+1}\left(k_{p}+\frac{F^{\prime}(p)}{2 k_{p}}(t-p)+O\left(k_{p}^{-1-2 \varepsilon}\right)\right) d t+\int_{n}^{x}\left(k_{n}+O\left(k_{n}^{-\varepsilon}\right)\right) d t= \\
=\sum_{p=x_{0}}^{n-1}\left(k_{p}+\frac{F^{\prime}(p)}{4 k_{p}}+O\left(k_{p}^{-1-2 \varepsilon}\right)\right)+k_{n}(x-n)+O\left(k_{n}^{-\varepsilon}\right)= \\
=\sum_{p=x_{0}}^{n-1}\left(\Delta(p)+O\left(k_{p}^{-1-2 \varepsilon}\right)\right)+k_{n}(x-n)+O\left(k_{n}^{-\varepsilon}\right)= \\
=\Theta(n)+k_{n}(x-n)+O\left(k_{n}^{-\varepsilon}\right) .
\end{aligned}
$$

Подставляя полученное выражение в формулу (6.3), найдем

$$
\begin{aligned}
\psi(x)= & \frac{s_{l}}{\sqrt[4]{F(x)}} \exp \left(i \int_{x_{0}}^{x} \sqrt{E+F(t)} d t\right)+ \\
& +\frac{t_{l}}{\sqrt[4]{F(x)}} \exp \left(-i \int_{x_{0}}^{x} \sqrt{E+F(t)} d t\right)+O\left(\frac{\left\|\mathbf{s}_{l}\right\|}{\sqrt{F(x)}}\right), \quad x \in I_{l},
\end{aligned}
$$



где

$$
\left(\begin{array}{l}
s_{l} \\
t_{l}
\end{array}\right)=\left(\begin{array}{ll}
1 & 0 \\
0 & i
\end{array}\right)\left(\begin{array}{l}
\hat{s}_{l} \\
\hat{t}_{l}
\end{array}\right)
$$

Учитывая соотношение (6.2), находим связь между $\mathbf{s}_{l}$ и $\mathbf{s}_{l+1}$ :

$$
\mathbf{s}_{l+1}=W_{l} \mathbf{s}_{l}, \quad W_{l}=\left(\begin{array}{cc}
1 & 0 \\
0 & i
\end{array}\right) \widehat{W}_{l}\left(\begin{array}{cc}
1 & 0 \\
0 & -i
\end{array}\right) .
$$

Матрица перехода $W_{l}$ может быть записана в виде

$$
W_{l}=e^{-i \Theta_{l} \sigma_{3}} S_{l} e^{i \Theta_{l} \sigma_{3}}
$$

где мы использовали обозначения, введенные после формулы (1.5). При этом из тождества (5.14) следует, что матрица $S_{l}$ удовлетворяет соотношению $\sigma_{1} \overline{S_{l}} \sigma_{1}=S_{l}$.

Заметим, что из леммы 2 можно вывести оценку

$$
|\psi(x)|=O\left(\frac{\left\|\mathbf{s}_{l}\right\|}{\sqrt[4]{F(x)}}\right), \quad x \in \tilde{i}_{l}
$$

Отсюда и из формулы (6.4) несложно получить формулу (1.6). Этим завершается доказательство теоремы 1.

\section{7. СПЕКТРАЛЬНЫЕ СВОЙСТВА УРАВНЕНИЯ ШРЕДИНГЕРА}

Для доказательства теоремы 2 воспользуемся результатами из статьи [8]. Необходимое нам утверждение может быть сформулировано следующим образом.

ТеОрема [8]. Пусть $M_{\mathrm{ac}}-$ множество спектральных параметров $E \in \mathbb{R}$, удовлетворяющих следующему условию: для любых решений $\psi_{1}, \psi_{2}$ уравнения (1.1) существуют постоянные $C_{7} u C_{8}$ такие, что

$$
0<C_{7} \leqslant \frac{\int_{0}^{x}\left|\psi_{1}(t)\right|^{2} d t}{\int_{0}^{x}\left|\psi_{2}(t)\right|^{2} d t} \leqslant C_{8}<\infty, \quad x>0 .
$$

Пусть $M_{\mathrm{ac}}=\mathbb{R}$, тогда спектр оператора $H$ абсолютно непрерывен и заполняет всю вещественную осъ.

Центральной в доказательстве теоремы 2 является следующая лемма.

ЛЕмма 4. Пусть выполнены оценки (1.7), $\mathbf{s}_{l}$ - решение системы (1.5). Тогда существуют постоянные $C_{10}$ и $C_{11}$ такие, что $0<C_{10} \leqslant\left\|\mathbf{s}_{l}\right\| \leqslant C_{11}<\infty$.

ДоказАтельство. Справедлива оценка

$$
\left\|W_{l}\right\|=1+O\left(r(l) \kappa(l) l^{-1 / 2}\right)+O(\varrho(l))=1+O\left(r(l) l^{(\varepsilon-2) / 2}\right)+O(\varrho(l)) .
$$


Отсюда и из условия (1.7) следует, что

$$
\begin{aligned}
\left\|\mathbf{s}_{l}\right\| & \leqslant \prod_{k=1}^{l-1}\left\|W_{k}\right\|\left\|\mathbf{s}_{1}\right\| \leqslant \prod_{k=1}^{l-1}\left(1+C_{41}|r(l)| l^{(\varepsilon-2) / 2}+C_{42} \varrho(l)\right)\left\|\mathbf{s}_{1}\right\| \leqslant C_{43}\left\|\mathbf{s}_{1}\right\|<\infty, \\
0<\left\|\mathbf{s}_{1}\right\| \leqslant & \prod_{k=1}^{l-1}\left\|W_{k}^{-1}\right\|\left\|\mathbf{s}_{l}\right\| \leqslant \prod_{k=1}^{l-1}\left(1+C_{44}|r(l)| l^{(\varepsilon-2) / 2}+C_{45} \varrho(l)\right)\left\|\mathbf{s}_{l}\right\| \leqslant C_{46}\left\|\mathbf{s}_{l}\right\|,
\end{aligned}
$$

где $C_{46}>0$.

ДоКАЗАТЕЛЬСТВо тЕОРЕмы 2. Из теоремы 1 следует, что для произвольного решения $\psi_{1}$ уравнения (1.1) справедлива оценка

$\int_{0}^{x}\left|\psi_{1}(t)\right|^{2} d t=C_{50}+\sum_{k=l_{0}}^{l-1} \frac{2 \pi}{F^{\prime}\left(\tilde{n}_{k}\right)}\left\|\mathbf{s}_{k}\right\|^{2}(1+o(1))+\frac{x-\tilde{n}_{l-1}}{\pi l}\left\|\mathbf{s}_{l}\right\|^{2}(1+o(1)), \quad x \in\left[\tilde{n}_{l-1}, \tilde{n}_{l}\right]$.

Отсюда, используя лемму 4, можно показать, что применима теорема из работы [8]. Этим завершается доказательство теоремы.

Благодарности. Автор выражает благодарность В. С. Буслаеву за многочисленные стимулирующие обсуждения и подбор литературы по данной тематике. Работа поддержана РФФИ (гранты № 05-01-01076 и № 05-01-01077).

\section{Список литературы}

[1] А. А. Пожарский. Алгебра и анализ. 2004. Т. 16. № 3. С. 171-200.

[2] М. В. Федорюк. Асимптотические методы для линейных обыкновенных дифференциальных уравнений. М.: Наука, 1983.

[3] М. В. Буслаева. Функц. анализ и его прилож. 1984. Т. 18. № 1. С. 65-66; В. С. Буслаев. ТМФ. 1984. Т. 58. С. 233-243; В. С. Буслаев, Л. А. Дмитриева. ТМФ. 1987. T. 73. С. 430-442; Алгебра и анализ. 1989. Т. 1. № 2. С. 1-29; J. Avron, L. Gunter, J. Zak. Solid State Commun. 1975. V. 16. № 2. P. 189-191; J. Avron, J. Zak. J. Math. Phys. 1977. V. 18. № 5. P. 918-921; A. Nenciu, G. Nenciu. J. Phys. A. 1981. V. 14. № 10. P. 2817-2827.

[4] А. А. Пожарский. ТМФ. 2000. Т. 123. С. 132-149; Алгебра и анализ. 2002. Т. 14. № 1. C. $158-193$.

[5] P. Exner. J. Math. Phys. 1995. V. 36. P. 4561-4570.

[6] V. S. Buslaev. Kronig-Penney electron in a homogeneous electric field. In: Differential Operators and Spectral Theory. M. Sh. Birman's 70th anniversary collection. Transl. Ser. 2. AMS Adv. Math. Sci. 1999. V. 189. Eds. V. Buslaev, M. Solomyak, D. Yafaev. Providence, RI: AMS, 1999. P. 45-57.

[7] F. Delyon, B. Simon, B. Souillard. Ann. Inst. H. Poincaré. Phys. Théor. 1985. V. 42. № 3. P. $283-309$

[8] D. J. Gilbert, D. B. Pearson. J. Math. Anal. Appl. 1987. V. 128. P. 30-56. 